\title{
Heart rate variability and respiratory muscle strenght in a stroke patient submitted to neurofeedback: A case report
}

\author{
Luana Karoline Castro Silva1, Barbara Galdino de Sousa ${ }^{1}$, Paulo Cezar Nascimento Filho², Florence Tupinambá \\ Duarte $^{2}$, José Rogério Santana ${ }^{3}$, Francisco Fleury Uchoa Santos Junior ${ }^{1,2}$
}

${ }^{1}$ Centro Universitário Estácio do Ceará - Fortaleza (CE), Brazil; ${ }^{2}$ Instituto Le Santé - Fortaleza (CE), Brazil; ${ }^{3}$ Universidade Federal do Ceará Fortaleza (CE), Brazil.

\section{ABSTRACT}

Background: Stroke is a neurological disease that causes sudden loss of brain functions. One of the most important symptoms is hemiparesis, which due to the weakness of the trunk promotes a decrease in diaphragmatic power and impairs pulmonary function. In addition to the respiratory system, stroke can lead to impairment of the cardiovascular system. This occurs when the Autonomic Nervous System is affected, increasing the risk of the development of Heart Rate Variability due to sympathetic hyperactivity, this condition may predict an unfavorable prognosis after stroke. Case presentation: The aim of this study was to evaluate the variability of heart rate and respiratory muscle strength in a 61-year-old female patient diagnosed with a stroke three months prior to the study submitted to Neurofeedback training. That's the first report of this kind in the literature. Cardiopulmonary evaluation was performed with a focus on heart rate and respiratory muscle strength before and after an intervention with five brain training sessions using the Neurofeedback BrainLink LITE. Conclusion: After therapy, significant alterations in the Delta wave increase and reduction of the Alpha wave were observed, also changes in the Autonomic Nervous System parameters were observed, with reduction of the sympathetic activity and increase of the parasympathetic activity, along with that, a reduction in the stress index was observed. The intervention was still positive in the improvement of respiratory parameters, with an increase in the overall inspiratory muscle strength index (S-Index) and peak inspiratory flow (PIF). It is suggested that Neurofeedback is efficient in recovering functions related to Autonomic Nervous System and respiratory system.

Keywords: Cardiorespiratory System; Autonomic Nervous System; Neurofeedback.

\section{BACKGROUND}

Stroke is defined as a neurological disease that causes sudden loss of brain functions with the fast development of clinical signs. ${ }^{(1)}$ The type of dysfunction and the consequences present after a stroke are determined by the region of the lesion in the brain and by the extension of the injury. ${ }^{(2)}$

The loss of motor functions is one of the major complaints after a stroke, one of the most important motor symptoms is the muscle weakness, which can affect the upper limb, trunk and lower limb of the hemibody contralateral to the injury. The trunk paresis can lead to postural dysfunctions, which keep the ribcage in an inspirational position, promoting a decrease in the activation of the abdominal muscles. In consequence, it leads to inefficiency of the respiratory muscles and pulmonary mechanics, subsequently, respiratory problems. (3) These changes promote a decrease in the values of the maximum pulmonary pressures related to respiration. ${ }^{(3,4)}$

Stroke can also lead to impairment of the cardiovascular system. This happens when the Autonomic Nervous System (ANS) is affected. ${ }^{(5,6)}$ The ANS is the part of the nervous system that controls involuntary functions of the organism, it's divided into sympathetic and parasympathetic subsystem. The sympathetic subsystem is activating in stress situations; while the parasympathetic predominates in recovery, during the state of greater stability of the body. $(5,7)$

After a stroke, when there is the presence of autonomic cardiovascular dysfunction, it's common to observe a sympathetic hyperactivity and parasympathetic dysfunction. When the autonomic functions areas related are affected, there may be a change in heart rate variability (HRV) ${ }^{(8,9)}$ In this view, the control of HRV can be a predictor in the prevention of recurrence of stroke. In the same way, the strength gain of the respiratory muscles may be important for the return of activities and greater social participation of the patient with stroke sequelae.

\section{CASE PRESENTATION}

A 61-year-old female with a diagnosis of Stroke occurring three months prior to the study. An evaluation of the sociodemographic variables was performed, and data from the cardiopulmonary physical examination was collected, focusing on the heart rate $(\mathrm{HR})$ and respiratory muscle strength, before and after an intervention with five brain training sessions using the Neurofeedback BrainLink LITE. 
The evaluation proceeded, in order, with the cerebral mapping through quantitative electroencephalography (qEEG), followed by the evaluation of HRV through $\mathrm{EmWave}^{\mathrm{TM}}$ (Hearthmath) and respiratory muscle strength with data of peak inspiratory flow (PIF) and muscle strength index (S-Index) evaluated through PowerBreathe (POWERbreathe Kinetic Series, HaB international, UK). The research protocol was structured as follows: on the first day, the variables were assessed and the first training was performed. The others four training sessions were performed in the others 2 weeks on intercalated days.

On the fifth and last day of training, a re-evaluation of the variables was executed. Each training lasted 15 minutes. All the trainings were performed with the Neurofeedback BrainLink LITE, which focuses on the training of the Fp1 area, related to the executive functions of the brain. The study was approved by the Research Ethics Committee involving human beings of the Estácio do Ceará University Center under protocol 2,908,211. In table 1, the data related to the activation of the ANS, that is, the values of the activation index of the Simpathetic Nervous System (SNS) and the Parassimpathetic Nervous System (PNS), of the mean heart rate (HR) and the stress index, resulting from the evaluation with EmWave and summarizes the values of S-Index and PIF obtained with PowerBreathe, before and after the intervention with NFB. The values of overall inspiratory muscle strength index (S-Index) and peak inspiratory flow (PIF) before and after NFB intervention are disposed in figure 1.

The table 2 and table 3, respectively, disposes the mean before and after intervention and its difference and $95 \%$ confidence interval $(\mathrm{Cl})$ of activation of brain waves with open eyes and closed eyes whereas the figure 2 shows the relative values (percentage of total EEG) for Delta waves and Alpha waves with eyes closed, open and on task, before and after NFB intervention.

Table 1. EmWave variables and S-Index and PIF values before and after NFB intervention

\begin{tabular}{lccl}
\hline Variable & $\begin{array}{c}\text { Before } \\
\text { intervention }\end{array}$ & $\begin{array}{c}\text { After } \\
\text { intervention }\end{array}$ & Difference \\
\hline HR Mean & $76 \mathrm{bpm}$ & $73 \mathrm{bpm}$ & $-3 \mathrm{bpm}$ \\
PNS Index & 3,51 & 5,12 & 1,61 \\
SNS Index & $-0,36$ & $-0,71$ & 0,35 \\
Stress Index & 4,4 & 3,6 & $-0,80$ \\
S-Index & 39,75 & 51,88 & 15,13 \\
PIF & 2,19 & 2,90 & 0,71 \\
\hline
\end{tabular}

Note: Heart rate (HR), Parassimpathetic Nervous System (PNS), Simpathetic Nervous System (SNS), Strength index (S-Index), Peak inspiratory flow (PIF).
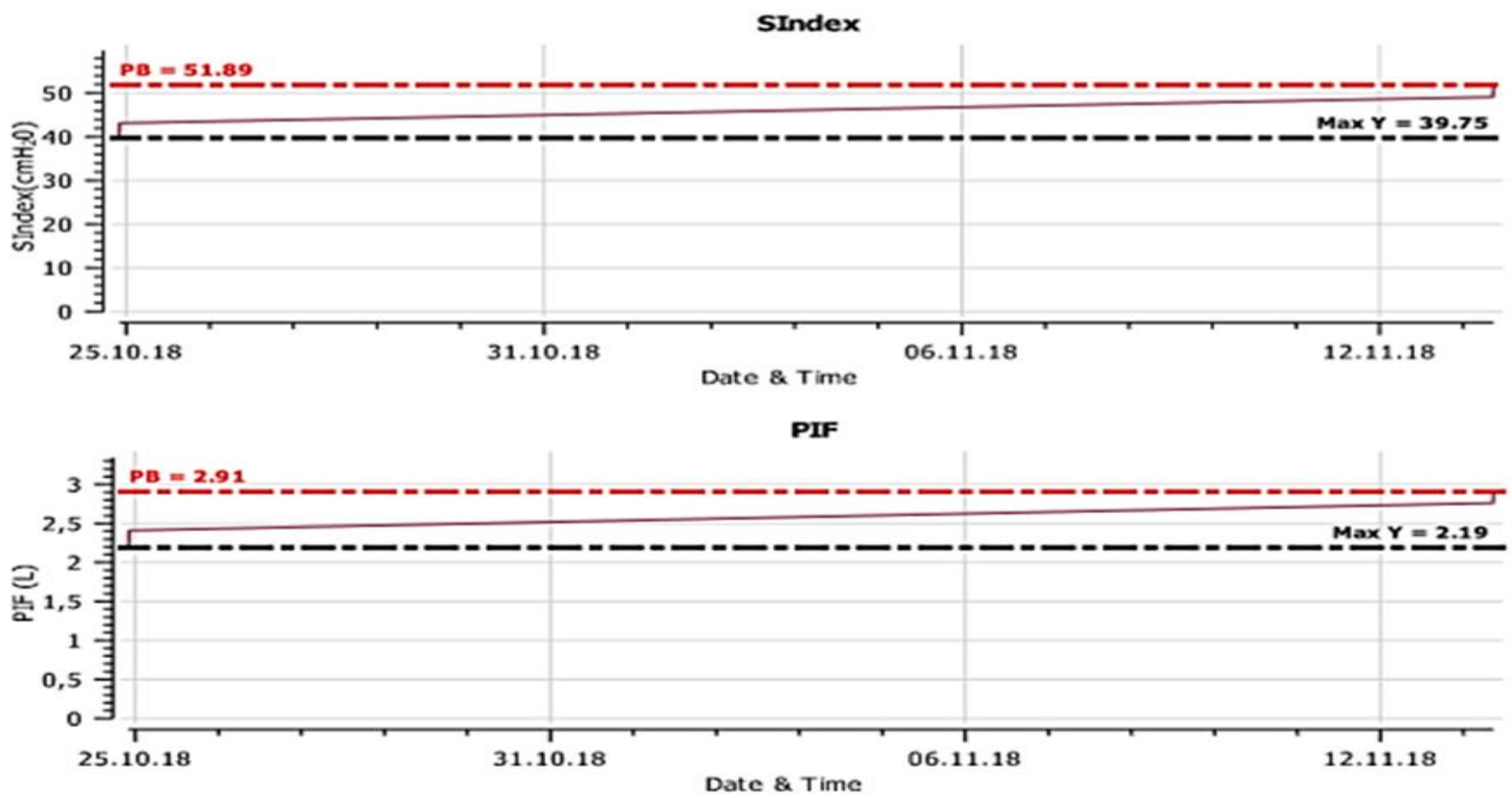

Figure 1. Values of overall inspiratory muscle strength index (S-Index) and peak inspiratory flow (PIF) before and after NFB intervention. 
Table 2. Mean difference and $95 \%$ confidence interval of brain waves activation with open eyes.

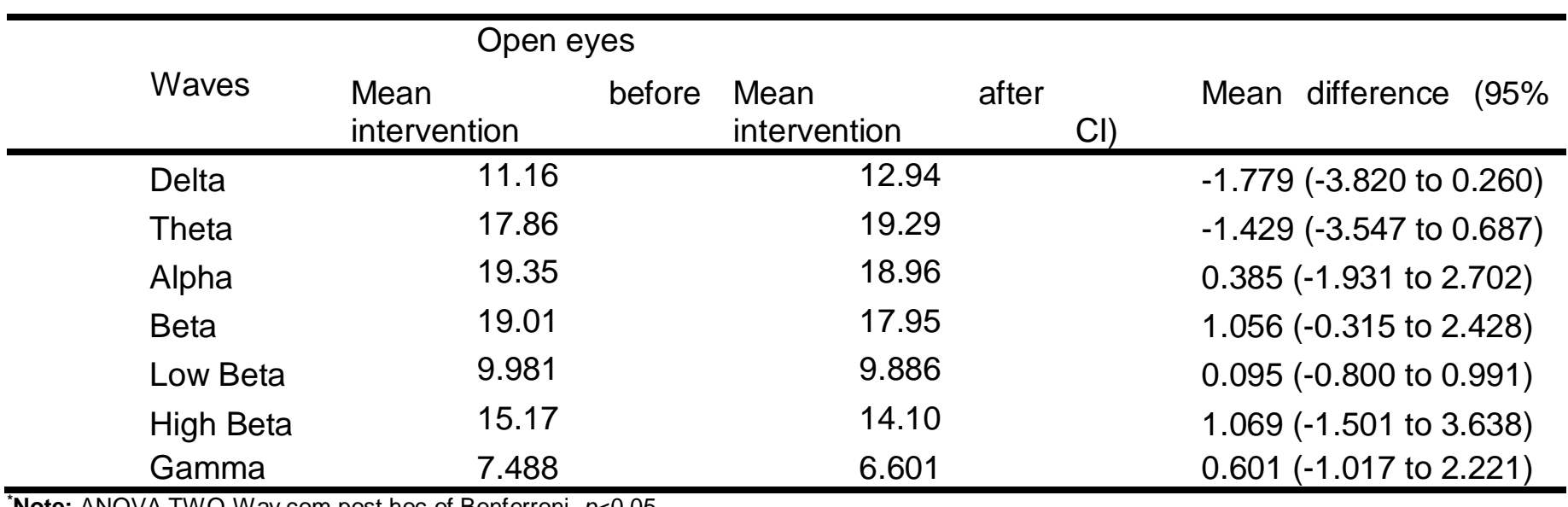

Note: ANOVA TWO-Way com post hoc of Bonferroni. $p<0,05$.

Table 3. Mean difference and 95\% confidence interval of brain waves activation with closed eyes.

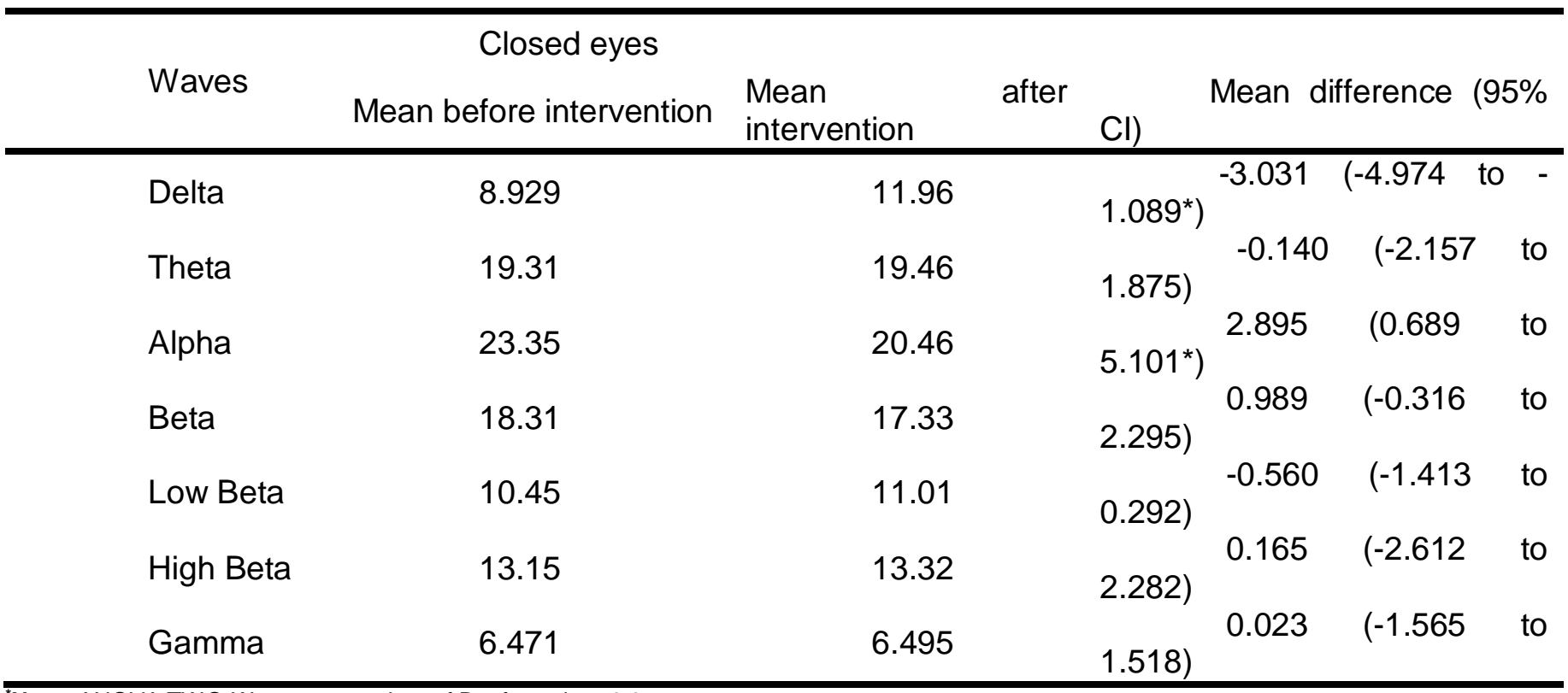

Note: ANOVA TWO-Way com post hoc of Bonferroni. $p<0,05$

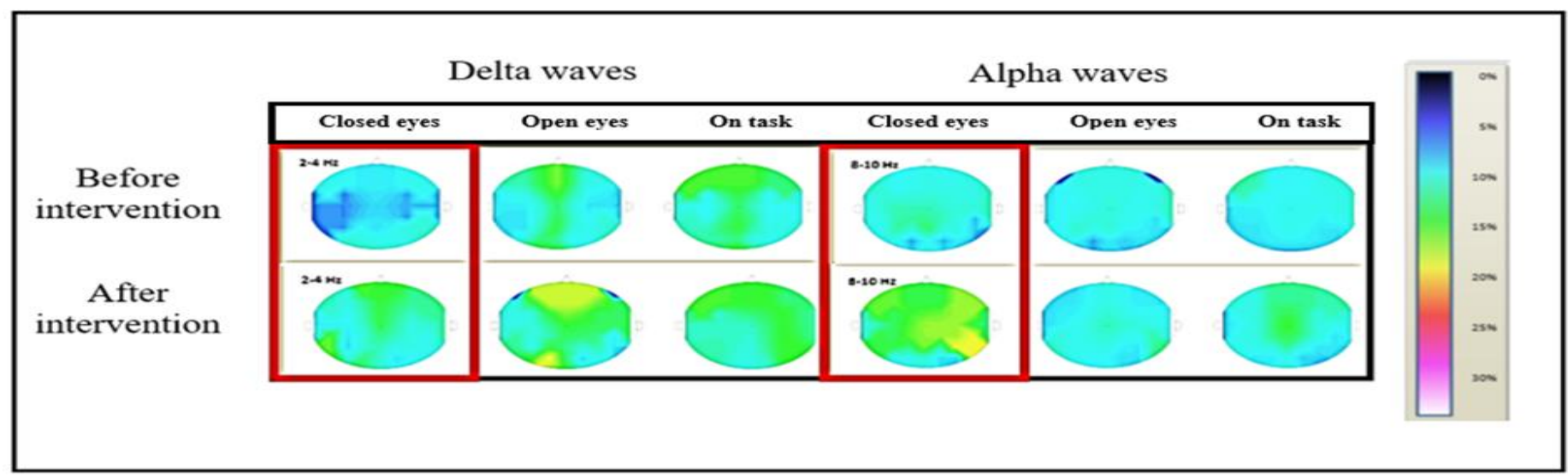

Figure 2. Relative values (percentage of total EEG) for Delta waves and Alpha waves with eyes closed, open and on task, before and after NFB intervention.

*Note: electroencephalography (EEG), Near Field Communication (NFC). 


\section{DISCUSSION}

This study questioned the applicability of five training sessions with Neurofeedback BrainLink LITE as therapy in the conditioning of the cardiorespiratory system. It was hypothesized that NFB training would be capable of performed, in part, by ANS, which provides afferent and efferent nerves to the heart. The increase in HR is a consequence of the greater action of the sympathetic pathway and the lower parasympathetic activity. Thus, the elevation of the parasympathetic modulation induces an electrical stability of the heart, whereas the high sympathetic activity increases the vulnerability of the heart and the risk of cardiovascular events. ${ }^{(10-13)}$

Interventions on HRV can work by increasing parasympathetic activation in the context of stress, and in response to this, a physiological mitigation of stress response is possible, supporting the parasympathetic activity to buffer the sympathetic reactions to stress. ${ }^{(14)}$

Others authors corroborates with our findings, where after an increase in parasympathetic activity there was an improvement in the patient's stress indexes. After NFB training, SNS activity decreased and PNS an increased, resulting in a reduction of stress and HRV..$^{(15,16)}$

In the mapping of the brain waves, a change was detected in the activation of Delta and Alpha waves. After the intervention, Delta was significantly increased during the evaluation with closed eyes, just as Alpha presented a relative reduction in the same evaluation (FIGURE 2). The Delta wave, considered a low frequency wave and a large amplitude $(0.5 \mathrm{~Hz}-4 \mathrm{~Hz})$, is related to deep sleep, to the point that Alfas waves $(7.5-12.5)$ are associated with the state of relaxation, attention and awareness. It is assumed that Deltas waves are associated with nonRapid Eyes Movement (N-REM) sleep. ${ }^{(17)}$ Exist a relationship between ANS and sleep states that shows that during REM sleep the SNS is more activated than the PNS. The opposite occurs during N-REM sleep, where there is a lower activation of SNS and greater activation of the PNS. ${ }^{(18)}$

In addition, there is a relationship with the results found in this study, where there was a decrease in SNS and a significant increase in Deltas waves, corroborating with the physiological processes already described in the literature. It's reported that sleep is also an important modulator of cardiovascular function, where there is a temporal association between physiological sleep and the occurrence of vascular events, cardiac arrhythmias and sudden death. It indicates that there is a possible correlation between primary sleep abnormalities and metabolic and cardiovascular diseases such as hypertension, atherosclerosis, stroke, heart failure, cardiac arrhythmias, sudden death, obesity, and metabolic syndrome. ${ }^{(19)}$ Furthermore, to these promoting changes in the brain areas responsible for ANS and thus bring HRV adjustments, as well as being able to promote respiratory muscle strength improvement. The control of the cardiovascular system is parameters, it was possible to observe significant changes in Delta and Alpha brainwaves patterns.

These waves are important in sleep quality since the Delta wave is related to deep sleep (N-REM), by the existing relationship between PNS with the generation of $\mathrm{N}$-REM sleep, it's questioned whether the change in the parasympathetic system is related to the increase found in Deltas waves. Besides this, studies that relate respiratory muscle strength to the results of an NFB training protocol have not been described in the literature, yet. This study, however, presents this hypothesis. As a result, a positive difference was found with an increase in inspiratory variables after the application of five NFB training.

\section{CONCLUSION}

Finally, the applicability of five training sessions with Neurofeedback training generated an increase in variables related to respiratory function: S-Index and PIF. As expected, there was a change in the pattern of activation of the ANS, nothing that the training was sufficient to change the mean heart rate, decrease the SNS index and increase the PNS and reduce the stress index. Finally, it is concluded that NFB training may be a way in stroke therapy of conditions related to ANS and cardiorespiratory function. In view of the result being related to a single case, it is proposed the need to reproduce this research in a larger scale, for a more statistical approach.

AUTHORS' CONTRIBUTIONS: LKCS and BGS contributed to the elaboration of the design, development of the study and data acquisition. PCNF and FTD contributed to article design and data tabulation. JRS contributed to the critical review, correction and approval of the final version. FFUSJ contributed to the elaboration of the study design and development and data analysis, and approval of the final version.

FINANCIAL SUPPORT: The authors have not received any financial support for the research, authorship and/or publication of this article.

CONFLICT OF INTEREST: The authors declare that there was no conflict of interests.

\section{REFERENCES}

1. World Health Organization. (2006). WHO STEPS stroke manual: the WHO STEPwise approach to stroke surveillance. World Health Organization.

2. Machado A. Neuroanatomia functional. 3th ed. Rio de Janeiro: Atheneu; 2013. 
3. Meneghetti $\mathrm{CHZ}$, Figueiredo VE, Guedes AV, Batistela ACT. Avaliação da Força Muscular Respiratória em Indivíduos Acometidos por Acidente Vascular Cerebral. Rev. Neurocienc. 2011;19(1):5660.

4. Schuster RC. Correlação entre Disfunções Motoras e Respiratórias no AVC. Rev Neurocienc. 2011;19(4):587-588.

5. Makivić B, Nikić M, Willis MS. Heart Rate Variability (HRV) as a Tool for Diagnostic and Monitoring Performance in Sport and Physical Activities. JEP online. 2013;16(3):103-131.

6. Rincon F, Dhamoon M, Moon Y, Paik MC, BodenAlbala B, Homma S, et al. Stroke location and association with fatal cardiac outcomes: Northern Manhattan Study (NOMAS). Stroke. 2008;39(9):2425-31.

7. Hall J, Guyton H. Textbook of Medical Physiology, 12th ed. Saunders -Elsevier, 2011.

8. Al-Qudah ZA, Yacoub HA, Souayah N. Disorders of the Autonomic Nervous System after Hemispheric Cerebrovascular Disorders: an update. Journal of Vascular and Interventional Neurology. 2015;8(4):43-52.

9. Hilz MJ, Moeller S, Akhundova A, Marthol H, Pauli E, De Fina $P$, et al. High NIHSS values predict impairment of cardiovascular autonomic control. Stroke. 2011;42:1528-33.

10. Kaikkonen $P$, Nummela $A$, Rusko $H$. Heart rate variability dynamics during early recovery after different endurance exercises. European Journal of Applied Physiology. 2007;102:79-86.

11. Burnley M, Doust JH, Jones AM. Time required for the restoration of normal heavy exercise VO2 kinetics following prior heavy exercise. Journal of Applied Physiology. 2006;101:1320-1327.
12. Casties JF, Mottet T, Le Gallais D. Non-Linear Analyses of Heart Rate Variability During Heavy Exercise and Recovery in Cyclists. International Journal of Sports Medicine. 2006;27:780-785.

13. Acharya UR, Fausta $\mathrm{O}$, Kannathala N, Chuaa T, Laxminarayanb $S$. Non-linear analysis of EEG signals at various sleep stages. Computer Methods and Programs in Biomedicine. 2005;80:37-45.

14. Hallman D, Olsson E, Von Scheele B, Melin L, Lyskov E. Effects of heart rate variability biofeedback in subjects with stress-related chronic neck pain: A pilot study. Applied Psychophysiology and Biofeedback. 2011;36:71-80.

15. Whited A, Larkin KT, Whited M. Effectiveness of emWave Biofeedback in Improving Heart Rate Variability Reactivity to and Recovery from Stress. Appl sychophysiol Biofeedback. 2014;39(2):75-88.

16. Chen S, Sun P, Wang S, Lin G, Wang T. Effects of heart rate variability biofeedback on cardiovascular responses and autonomic sympathovagal modulation following stressor tasks in prehypertensives. Journal of human hypertension. 2016;30(2):105.

17. Jansen JM, Lopes AJ, Jansen U, Capone D, Maeda TY, Noronha A, Magalhães G. Medicina da noite: da cronobiologia à prática clínica. Cad. Saúde Pública. 2008; 24(10):2452-2455.

18. Jacomini LCL, Silva NA. Disautonomia: um conceito emergente na síndrome da fibromialgia. Revista Brasileira de Reumatologia. 2007;47(5):354-361.

19. Wolk R, Gami AS, Garcia-Touchard A, Somers VK. Sleep and cardiovascular disease. Curr Probl Cardiol. 2005;7(2):625-662. 